\title{
Corrigendum: An exactly solvable model for the integrability-chaos transition in rough quantum billiards
}

Maxim Olshanii, Kurt Jacobs, Marcos Rigol, Vanja Dunjko, Harry Kennard \& Vladimir A. Yurovsky

Nature Communications 3:641 doi: 10.1038/ncomms1653 (2012); Published 24 Jan 2012; Updated 5 Feb 2013

The funding information for this Article was not fully acknowledged. The Acknowledgements should have read:

We are grateful to F. Werner and D. Cohen for enlightening discussions on the subject. Supported by the Office of Naval Research grant N00014-09-1-0502 (M.O. and V.D.) and N00014-09-1-0966 (M.R.), and the National Science Foundation grants PHY-1019197 (M.O. and V.D.) and PHY-0902906 (K.J.). 